\title{
Stable MSAP Markers for the Distinction of Vitis vinifera cv Pinot Noir Clones
}

\author{
Juan Ocaña $\cdot$ Bernard Walter $\cdot$ Paul Schellenbaum
}

Published online: 31 May 2013

(C) Springer Science+Business Media New York 2013

\begin{abstract}
Grapevine is one of the most economically important fruit crops. Molecular markers have been used to study grapevine diversity. For instance, simple sequence repeats are a powerful tool for identification of grapevine cultivars, while amplified fragment length polymorphisms have shown their usefulness in intra-varietal diversity studies. Other techniques such as sequence-specific amplified polymorphism are based on the presence of mobile elements in the genome, but their detection lies upon their activity. Relevant attention has been drawn toward epigenetic sources of variation. In this study, a set of Vitis vinifera cv Pinot noir clones were analyzed using the methylation-sensitive amplified polymorphism technique with isoschizomers MspI and HpaII. Nine out of fourteen selective primer combinations were informative and generated two types of polymorphic fragments which were categorized as "stable" and "unstable." In total, 23 stable fragments were detected and they discriminated $92.5 \%$ of the studied clones. Detected stable polymorphisms were either common to several clones, restricted to a few clones or unique to a single clone. The identification of these stable epigenetic markers will be useful in clonal diversity studies. We highlight the relevance of stable epigenetic variation in $V$. vinifera clones and analyze at which level these markers could be applicable for the development of forthright techniques for clonal distinction.
\end{abstract}

J. Ocaña $\cdot$ B. Walter $\cdot$ P. Schellenbaum $(\bowtie)$

Laboratoire Vigne, Biotechnologies et Environnement,

Université de Haute Alsace, 33 rue de Herrlisheim,

68008 Colmar, France

e-mail: paul.schellenbaum@uha.fr
Keywords Epigenetic - DNA methylation - Grapevine · Pinot noir - Clonal variation - Stable polymorphism · Unstable polymorphism · PIC

\section{Introduction}

Most of the grapevine varieties-called cépages-grown for the production of high-quality wines belong to the European species Vitis vinifera. Among them, Pinot is one of the most diverse and is used for producing famous wines particularly in France (Burgundy and Champagne), Germany and Switzerland. In the variety Pinot, the color of the berry is white, gray or black leading to the distinction of Pinot blanc, Pinot gris and Pinot noir, respectively. Pinot noir provides a great pool of clonal phenotypes displaying plasticity in canopy growth, cluster architecture, fruit yield and maturity.

Grapevine is commonly reproduced by vegetative propagation cycles during which phenotypic differences can accumulate giving rise to clonal diversity. Two possible origins for this diversity have been proposed depending on the initial germplasm from which the variety was selected from. A polyzygotic origin has been proposed when more than one seedling, with homogeneous phenotypic characters, gave rise to one variety [1]. A monozygotic origin probably is the most frequent: In this case, a single seedling gave rise to one variety, and phenotypic variations between clones are the result of somatic modifications spontaneously occurring during vegetative propagation and conservation. Somatic variation can be induced by genetic or by epigenetic determinants. Various types of genetic modifications have been shown to be correlated with clonal diversity in grapevine varieties: point mutations, indels, illegitimate recombination, variable number 
of repeats in microsatellite sequences, variation in ploidy, dynamic of mobile elements [2, 3]. In a genome-wide analysis of polymorphism among 3 Pinot noir clones, Carrier et al. [3] found three types of polymorphism: single nucleotide polymorphisms (SNPs), indels and mobile elements. The reported polymorphism rate was quite low for SNPs (1.6/Mb) and indels (5.1/Mb), while the major cause of somatic polymorphism was insertion polymorphism by mobile elements (35.2/Mb). In previous studies, simple sequence repeats (SSRs) have been used for grapevine cultivar identification and for studying genetic diversity [4-6]. Even though their efficiency could be limited at the clonal level, SSRs are useful for the management of grapevine germplasm collections and nurseries [7, 8]. Amplified fragment length polymorphisms (AFLPs) have proven their efficiency in discriminating grape accessions and biotypes from the same cultivar, although their ability to generate many molecular polymorphisms may depend on the cultivar/variety analyzed [7, 9-11]. Nevertheless, other methods such as sequence-specific amplified polymorphism (S-SAP) revealed polymorphism among Pinot noir clones with universal retrotransposon-based primers [12] or primers based on Gret-1, Copia-10, Gypsy-19 and Cauliv-1 transposable elements [3], but not with Vine-1based primers $[9,13]$.

Differences between clones can also result from epigenetic modifications in response to the environment $[14,15]$. Epigenetic pathways (DNA methylation, histone modifications, positioning of nucleosomes, and small RNAs) are important components of plant growth and development. In the genome of higher plants, 5-methyl cytosine methylation is frequent and plays an important role in defense against activation and movement of transposable elements and regulation of gene expression.

In order to adapt to environmental stress and predation by pathogens and herbivores, one plant genotype often displays alternative phenotypes under different conditions. Phenotypic plasticity is sometimes equated with epigenetic variation [16]. Most examples of phenotypic plasticity are reversible, but other cases document transgenerational memory of stressful environments passed from parents to their unstressed progeny. Stable, inherited epigenetic variation might play an important role in the adaptation of plants and evolution [16].

In plant material where genetic variation is reduced as much as possible (apomictic lineages or clones generated by vegetative propagation), changes in genomic methylation patterns have been reported [17-20]. Efficient tools for distinguishing grapevine clones are highly needed to ensure quality management, track commercial propagation material and analyze germplasm collections [5, 8].

In the present study, a set of 40 Pinot noir clones which included 33 clones registered in France were analyzed using the methylation-sensitive amplified polymorphism (MSAP) technique. Our study aimed at the distinction of Pinot noir clones making use of their epigenetic differences. We provide an insight into the stability of these epigenetic differences and attempt to understand the mechanisms behind clonal variation in Pinot noir. Once identified, stable epigenetic markers can be used (1) to evaluate the clonal diversity during selection and conservation of genetic resources and (2) to guarantee the authenticity of commercialized clones which is crucial for nurserymen and grapevine growers in order to preserve specific quality standards.

\section{Materials and Methods}

\section{Plant Materials}

A total of 40 clones of $V$. vinifera Pinot noir were used in this study: 33 clones registered in the catalogue of grapevine varieties cultivated in France and 7 recently selected not yet registered clones from ATVB (Association Technique Viticole de Bourgogne, Beaune, France) and Pépinières Guillaume (Charcenne, France). All clones were analyzed using 2 or 5 replicates (Table 1). In most cases, 2 plants from a given clone were sampled in a given field plot or in different plots. For clones PN115, PN459, PN583, PN743 and PN777, 5 plants were sampled from different plots. In addition, $V$. vinifera Syrah clone 174 and $V$ vinifera Sangiovese clone 10-39 were also included. Young leaves were sampled from grapevines in multiplication field plots.

\section{DNA Extraction}

A modified CTAB-based protocol [21] was used, with modifications in the extraction buffer only. This extraction buffer contained $2 \%$ CTAB, $100 \mathrm{mM}$ Tris, $20 \mathrm{mM}$ EDTA, $1.4 \mathrm{M} \mathrm{NaCl}, 2 \%$ 2-mercaptoethanol and $3 \%$ PVP-40. For each extraction, $50 \mathrm{mg}$ of young leaves were frozen in liquid nitrogen and crushed using a mixer mill (MM200, $\operatorname{Retsch}^{\circledR}$, Haan, Germany). DNA samples were then re-purified using the NucleoSpin ${ }^{\circledR}$ II Purification Kit (Macherey-Nagel, Düren, Germany) following the manufacturer's instructions. DNA concentrations were quantified using the Qubit $^{\mathrm{TM}}$ quantitation platform (Life Technologies SAS, Saint Aubin, France). DNA quality was verified by electrophoresis on $1.2 \%$ agarose gel.

Methylation-Sensitive Amplified Polymorphism (MSAP)

MSAP protocol and PCR amplification conditions were the same as described by Schellenbaum et al. [19]. For each 
Table 1 Pinot noir clones analyzed by MSAP

\begin{tabular}{|c|c|c|c|}
\hline Clone number & Origin & $\begin{array}{l}\text { Registration } \\
\text { year }\end{array}$ & $\begin{array}{l}\text { Number of } \\
\text { tested plants }\end{array}$ \\
\hline PN 111 & Côte d'Or & 1971 & 2 \\
\hline PN 112 & Côte d'Or & 1971 & 2 \\
\hline PN 113 & Côte d'Or & 1971 & 2 \\
\hline PN 114 & Côte d'Or & 1971 & 2 \\
\hline PN 115 & Côte d'Or & 1971 & 5 \\
\hline PN 164 & Côte d'Or & 1972 & 2 \\
\hline PN 165 & Côte d'Or & 1972 & 2 \\
\hline PN 236 & Côte d'Or & 1973 & 2 \\
\hline PN 292 & Jura & 1973 & 2 \\
\hline PN 374 & Jura & 1975 & 2 \\
\hline PN 375 & Saône-et-Loire & 1975 & 2 \\
\hline PN 386 & Marne & 1975 & 2 \\
\hline PN 388 & Marne & 1975 & 2 \\
\hline PN 389 & Marne & 1975 & 2 \\
\hline PN 459 & Jura & 1976 & 5 \\
\hline PN 461 & Côte d'Or & 1976 & 2 \\
\hline PN 462 & Côte d'Or & 1976 & 2 \\
\hline PN 521 & Marne & 1976 & 2 \\
\hline PN 528 & Not defined & 1976 & 2 \\
\hline PN 583 & Côte d'Or & 1978 & 5 \\
\hline PN 665 & Marne & 1980 & 2 \\
\hline PN 666 & Marne & 1980 & 2 \\
\hline PN 667 & Côte d'Or & 1980 & 2 \\
\hline PN 668 & Marne & 1980 & 2 \\
\hline PN 743 & Marne & 1981 & 5 \\
\hline PN 777 & Côte d'Or & 1981 & 5 \\
\hline PN 778 & Côte d'Or & 1981 & 2 \\
\hline PN 780 & Marne & 1981 & 2 \\
\hline PN 828 & Côte d'Or & 1985 & 2 \\
\hline PN 870 & Marne & 1986 & 2 \\
\hline PN 871 & Marne & 1988 & 2 \\
\hline PN 927 & Cher & 1988 & 2 \\
\hline PN 943 & Côte d'Or & 1989 & 2 \\
\hline $\mathrm{F} 1$ & Côte d'Or & & 2 \\
\hline TF1 & Côte d'Or & & 2 \\
\hline TF2 & Côte d'Or & & 2 \\
\hline PD79 & Côte d'Or & & 2 \\
\hline G10 & Côte d'Or & & 2 \\
\hline G2-13 & Côte d'Or & & 2 \\
\hline GL58 & Côte d'Or & & 2 \\
\hline
\end{tabular}

The number of plants analyzed for each clone is indicated. The geographical origins of clones and registration year were taken from the catalogue of Vines cultivated in France (IFV)

sample, between 200 and $400 \mathrm{ng}$ of genomic DNA were digested-ligated at $37{ }^{\circ} \mathrm{C}$ for $3 \mathrm{~h}$ in a $30 \mu \mathrm{l}$ reaction mix containing: $5 \mathrm{U}$ of either MspI or HpaII, $5 \mathrm{U}$ of EcoRI (New England BioLabs, Frankfurt am Main, Germany),
50 pmol of MspI-HpaII adapters (HM-A1 and HM-A2, Table 2) and 5 pmol of EcoRI adapters (Eco-A1 and EcoA2, Table 2), $1 \mathrm{U}$ of T4 DNA ligase, $1 \mathrm{mM}$ of ATP and $75 \mathrm{ng} / \mu \mathrm{l}$ of BSA. Digestion-ligation products were diluted $5 \times$ and $4 \mu \mathrm{l}$ was subsequently added to a preamplification mix containing $2.5 \mathrm{pmol}$ of each preamplification primer (E01 and HM0, Table 2), $1 \times$ PCR buffer, $0.2 \mathrm{mM}$ of dNTPs and $0.5 \mathrm{U}$ of Taq polymerase (Life Technologies SAS, Saint Aubin, France). Preamplification PCR conditions consisted in $2 \mathrm{~min}$ at $75^{\circ} \mathrm{C} ; 20$ cycles of $20 \mathrm{~s}$ at $94{ }^{\circ} \mathrm{C}, 30 \mathrm{~s}$ at $56{ }^{\circ} \mathrm{C}, 2 \mathrm{~min}$ at $72{ }^{\circ} \mathrm{C}$; followed by $30 \mathrm{~min}$ at $60{ }^{\circ} \mathrm{C}$.

Preamplification products were diluted 10 times and used for selective amplification of fragments using each one of the fourteen selective primer combinations (Table 2). Each selective amplification reaction mix contained $4 \mu \mathrm{l}$ of diluted preamplification product, $0.2 \mathrm{mM}$ of dNTPs, 5 pmol of MspI/HpaII primer, 1.5 pmol of fluorescently labeled EcoRI primer (IDT, Leuven, Belgium) and $0.5 \mathrm{U}$ of Taq polymerase (Life Technologies SAS, Saint Aubin, France) in $1 \times$ PCR buffer. For selective amplification, touchdown PCR conditions were as follows: 2 min at $94{ }^{\circ} \mathrm{C} ; 10$ cycles of $20 \mathrm{~s}$ at $94{ }^{\circ} \mathrm{C}, 30 \mathrm{~s}$ at $66^{\circ} \mathrm{C}$ decreasing by $1{ }^{\circ} \mathrm{C}$ per cycle, $2 \mathrm{~min}$ at $72{ }^{\circ} \mathrm{C}$; followed by 20 cycles of $20 \mathrm{~s}$ at $94{ }^{\circ} \mathrm{C}, 30 \mathrm{~s}$ at $56{ }^{\circ} \mathrm{C}, 2 \mathrm{~min}$ at $72{ }^{\circ} \mathrm{C}$; followed by $30 \mathrm{~min}$ at $60{ }^{\circ} \mathrm{C}$. Amplified fragments were separated in a $6.5 \%$ polyacrylamide gel and visualized using a $\mathrm{Li}_{\text {-Cor }}{ }^{\circledR} 4200 \mathrm{IR}^{2}$ sequencer (Welience, Dijon, France).

Both MspI and HpaII enzymes recognize and excise DNA sequences at CCGG restriction sites, but with different digestion sensitivity to cytosine methylation. MspI cleaves DNA when no cytosine is methylated or when the internal $\mathrm{C}$ is methylated $\left(\mathrm{C}^{\mathrm{m}} \mathrm{CGG}\right)$ on one or both DNA strands. HpaII cleaves in the case of no cytosine methylation or in the case of hemimethylation of the external ( $\left.{ }^{\mathrm{m}} \mathrm{CCGG}\right)$ or internal cytosine ( $\left.\mathrm{C}^{\mathrm{m}} \mathrm{CGG}\right)$ [22].

\section{MSAP Fragment Analysis}

Amplified fragments were scored using Saga ${ }^{\text {MX }}$ AFLP $^{\circledR}$ Software (Lincoln NE, USA). All fragments were individually counted as either present $(+)$ or absent $(-)$ and verified by two different users before generation of data matrixes for all tested primer combinations. Only visible and scorable bands throughout all analyzed gels for each primer combination were taken into account, and weak bands were eliminated. All replicates were included in the analysis to show the reproducibility of each marker.

Due to the nature of the identified polymorphisms, they were classified into two categories further used in this article: "stable polymorphisms" and "unstable polymorphisms." Stable polymorphisms correspond to fragments 
constantly either present or absent in all individual plants belonging to a single clone (Fig. 1), and unstable polymorphisms are fragments sometimes present and sometimes absent in different plant extracts from a given clone (Fig. 2).

Hierarchical Clustering Analysis and MSAP Marker Effectiveness

All generated marker data were transformed into a binary system coded as either present (1) or absent (0). Identified polymorphic markers were used to calculate epigenetic dissimilarities among Pinot noir clones by the unweighted pair group method with arithmetic mean algorithm (UPGMA) method using the R commander package [23].

In addition, the polymorphism information content (PIC) values were calculated to estimate the variability detected by MSAP markers and to quantify their usefulness in clonal distinction. PIC values were calculated for each primer combination using the formula as described by Botstein et al. [24],

$\mathrm{PIC}=1-\left(\sum_{i}^{n} P i^{2}\right)-2 \sum_{i}^{n-1} \sum_{j}^{n} P i^{2} P j^{2}$

where $P i$ and $P j$ represent the frequencies of the $i$ th et $j$ th alleles, respectively.

The percentage of stable polymorphisms was also calculated for all informative combinations $(100 \times$ number of polymorphic fragments/total number of MSAP fragments).

\section{Results}

MSAP Analysis of Pinot Noir Clones

The epigenetic profiles of $40 \mathrm{~V}$. vinifera Pinot noir clones were generated using the MSAP technique. A $V$. vinifera Syrah clone and a $V$. vinifera Sangiovese were included for comparison.

In total, 14 MSAP selective primer combinations were used (Table 2). Five combinations generated exclusively non-discriminating monomorphic markers (data not shown), while 9 combinations (further referred to as "informative combinations") produced both monomorphic and polymorphic markers. Monomorphic markers are MSAP fragments present in all clone samples, whereas polymorphic markers are MSAP fragments present or absent in at least one sample. The 9 informative combinations produced a total of 346 fragments after amplification of digested DNA using endonucleases EcoRI/MspI, further mentioned as "MspI fragments," whereas a total of 326 fragments were obtained using endonucleases EcoRI/ HpaII, further referred to as "HpaII fragments." Altogether, the informative combinations generated, on average, $38 \mathrm{MspI}$ and $36 \mathrm{HpaII}$ fragments per primer combination, ranging from 52 both $M s p I$ and HpaII with E32/HM35 to $22 \mathrm{MspI}$ and $23 \mathrm{HpaII}$ with E42/HM84 (Table 3). As a result of sample analysis by all informative primer combinations, a total of about 66,500 fragments were scored.

Table 2 MSAP primer combinations

\begin{tabular}{|c|c|}
\hline \multicolumn{2}{|l|}{ Ligation (EcoRI and $H p a \mathrm{II} / M s p \mathrm{I}$ adapters) } \\
\hline Eco-A1 = 5'-CTCGTAGACTGCGTACC-3' & HM-A1 = 5'-GATCATGAGTCCTGCT- $3^{\prime}$ \\
\hline Eco-A2 $=5^{\prime}$-AATTGGTACGCAGTCTAC- $3^{\prime}$ & HM-A2 $=5^{\prime}$-CGAGCAGGACTCATGA- $3^{\prime}$ \\
\hline \multicolumn{2}{|l|}{ Preamplification } \\
\hline $\mathrm{E} 01=5^{\prime}$-GACTGCGTACCAATTCA-3' & HM0 $=5^{\prime}$-ATCATGAGTCCTGCTCGG-3' \\
\hline \multicolumn{2}{|l|}{ Selective amplification } \\
\hline $\mathrm{E} 32=5^{\prime}$-GACTGCGTACCAATTCAAC-3' & HM35 $=5^{\prime}$-ATCATGAGTCCTGCTCGGACA- $3^{\prime}$ \\
\hline $\mathrm{E} 32=5^{\prime}-\mathrm{GACTGCGTACCAATTCAAC-3^{ \prime }}$ & HM70 $=5^{\prime}$-ATCATGAGTCCTGCTCGGGCT- $3^{\prime}$ \\
\hline E33 $=5^{\prime}$-GACTGCGTACCAATTCAAG-3' & HM46 $=5^{\prime}$-ATCATGAGTCCTGCTCGGATT- $3^{\prime}$ \\
\hline $\mathrm{E} 34=5^{\prime}-\mathrm{GACTGCGTACCAATTCAAT-3^{ \prime }}$ & HM49 $=5^{\prime}$-ATCATGAGTCCTGCTCGGCAG- $3^{\prime}$ \\
\hline $\mathrm{E} 35=5^{\prime}$-GACTGCGTACCAATTCACA-3' & HM62 = 5'-ATCATGAGTCCTGCTCGGCTT- $3^{\prime}$ \\
\hline $\mathrm{E} 35=5^{\prime}-\mathrm{GACTGCGTACCAATTCACA-3^{ \prime }}$ & HM70 $=5^{\prime}$-ATCATGAGTCCTGCTCGGGCT- $3^{\prime}$ \\
\hline $\mathrm{E} 38=5^{\prime}$-GACTGCGTACCAATTCACT- $3^{\prime}$ & HM70 $=5^{\prime}$-ATCATGAGTCCTGCTCGGGCT- $3^{\prime}$ \\
\hline $\mathrm{E} 39=5^{\prime}-\mathrm{GACTGCGTACCAATTCAGA-3^{ \prime }}$ & HM83 = 5'-ATCATGAGTCCTGCTCGGTCA- $3^{\prime}$ \\
\hline $\mathrm{E} 39=5^{\prime}-\mathrm{GACTGCGTACCAATTCAGA-3^{ \prime }}$ & HM46 = 5'-ATCATGAGTCCTGCTCGGATT- $3^{\prime}$ \\
\hline $\mathrm{E} 42=5^{\prime}-\mathrm{GACTGCGTACCAATTCAGT-3^{ \prime }}$ & HM84 = 5'-ATCATGAGTCCTGCTCGGTCC- $3^{\prime}$ \\
\hline $\mathrm{E} 45=5^{\prime}$-GACTGCGTACCAATTCATG-3' & HM34 $=5^{\prime}$-ATCATGAGTCCTGCTCGGAAT- $3^{\prime}$ \\
\hline $\mathrm{E} 45=5^{\prime}$-GACTGCGTACCAATTCATG-3' & HM62 = 5'-ATCATGAGTCCTGCTCGGCTT- $3^{\prime}$ \\
\hline $\mathrm{E} 45=5^{\prime}$-GACTGCGTACCAATTCATG-3' & HM83 $=5^{\prime}$-ATCATGAGTCCTGCTCGGTCA- $3^{\prime}$ \\
\hline $\mathrm{E} 46=5^{\prime}-\mathrm{GACTGCGTACCAATTCATT-} 3^{\prime}$ & HM84 $=5^{\prime}$-ATCATGAGTCCTGCTCGGTCC- $3^{\prime}$ \\
\hline
\end{tabular}




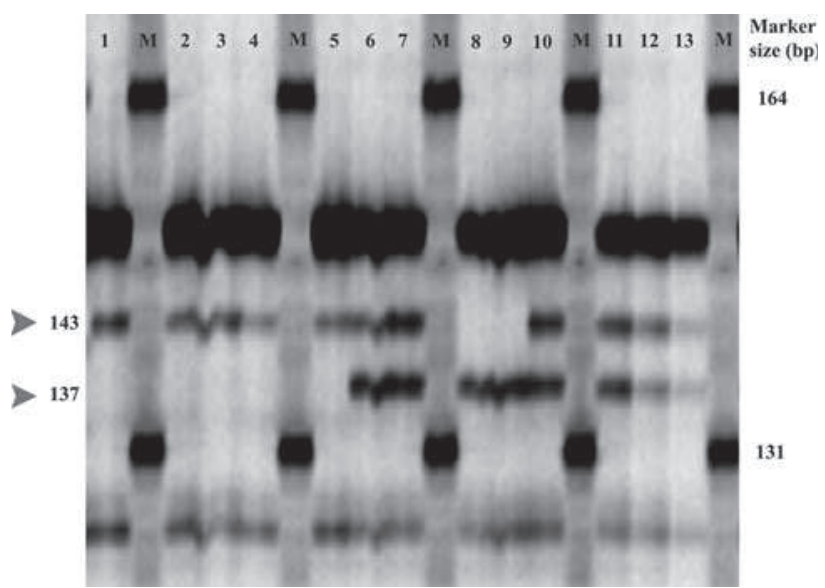

Fig. 1 Examples of partial MSAP profiles showing stable polymorphic markers. Thirteen partial MSAP profiles are shown in the gel image corresponding to 5 Pinot noir clones (PN777, PN828, PN943, F1 and TF1) using primer combination E42/HM84. Lanes: 1 (PN777plant 1), 2 (PN777-plant 2), 3 (PN777-plant 3), 4 (PN777-plant 4), 5 (PN777-plant 5), 6 (PN828-plant 1), 7 (PN828-plant 2), 8 (PN943plant 1), 9 (PN943-plant 2), 10 (F1-plant 1), 11 (F1-plant 2), 12 (TF1plant 1), 13 (TF1-plant 2), $M$ (700 bp DNA ladder). Marker at $137 \mathrm{bp}$ (arrow) distinguished clone PN777 (lanes 1-5) from the other 4 clones; clone PN943 (lanes 8, 9) is distinguished from the other clones by marker at 143 bp (arrow)

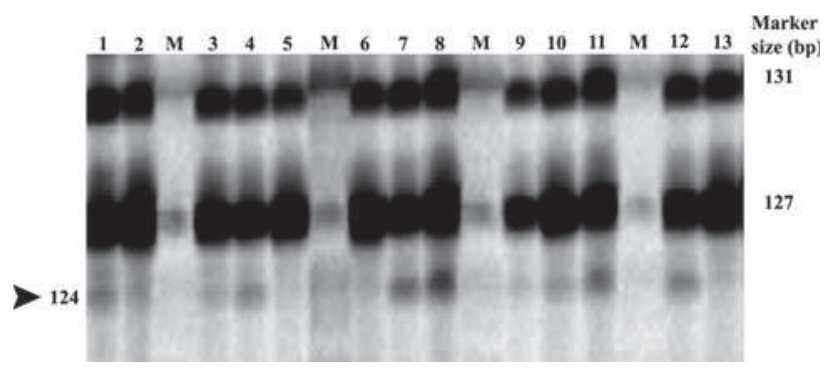

Fig. 2 Examples of partial MSAP profiles showing unstable polymorphic markers. Thirteen partial MSAP profiles are shown in the gel image corresponding to 5 Pinot Noir clones (PN113, PN114, PN115, PN165 and PN236). Lanes: 1 (PN113-plant 1), 2 (PN113-plant 2), 3 (PN114-plant 1), 4 (PN114-plant 2), 5 (PN115-plant 1), 6 (PN115plant 2), 7 (PN115-plant 3), 8 (PN115-plant 4), 9 (PN115-plant 5), 10 (PN165-plant 1), 11 (PN165-plant 2), 12 (PN236-plant 1), 13 (PN236-plant 2), $M$ (700 bp DNA ladder). One unstable polymorphic marker is indicated by the arrow (124 bp). Among 5 different plants corresponding to clone PN115 (lanes 5-9), this marker was present in 2 plants (lanes 7, 8) and absent in 3 plants (lanes 5, 6, 9). For clone PN236 (lanes 12, 13), the same marker was present in one plant (lane 12) and absent in the other plant (lane 13)

\section{Marker Stability Versus Marker Instability}

All detected MspI and HpaII polymorphic fragments were classified into two categories: "stable polymorphisms" and "unstable polymorphisms." Stable polymorphisms correspond to fragments either present or absent, repeatedly and without ambiguity, in all samples (different plants) of a given clone (Fig. 1). Unstable polymorphisms are fragments which are present in one or more samples and absent in at least one sample from a given clone (Fig. 2).

All monomorphic and polymorphic (stable and unstable) MspI and HpaII fragments were categorized as being "methylation-sensitive" (MS) and "methylation-insensitive" (MI) as described by Marfil et al. [25] (Table 3). MI fragments show no differences in EcoRI/HpaII and EcoRI/ MspI patterns. In contrast, MS fragments are amplified after digestion with either EcoRI/HpaII or EcoRI/MspI restriction enzymes.

In our study, MI polymorphic fragments were not detected by the MSAP technique. MI monomorphic fragments were the most abundant: $81 \%$ (281/346) of all MspI fragments and $86 \%(281 / 326)$ of all HpaII fragments. MS fragments were identified as either monomorphic (present as MspI fragments for all clones but absent as HpaII fragments or present as HpaII fragments for all clones but absent as MspI fragments) or polymorphic (stable or unstable) for all clones. The majority of MS monomorphic fragments were detected as being MspI fragments (61 in total) compared to HpaII fragments (13 in total) (Table 3).

Polymorphic stable fragments were mostly amplified as HpaII fragments (23) and a few as MspI fragments (2). The number of stable polymorphic fragments varied depending on the primer combination used, ranging from 1 (combinations E32/HM70 and E39/HM46) to 7 (combination E35/ HM70) (Table 3).

In the case of unstable polymorphic fragments, a comparable distribution was observed as for stable polymorphic fragments, displaying a higher number of HpaII fragments (9) compared to $M s p I$ fragments (2). The number of $M s p I$ and HpaII unstable fragments detected per combination fluctuated between 0 (combinations E32/HM35, E42/HM84, E45/ HM34) and 3 (combination E45/HM62) (Table 3).

Detected unstable polymorphic fragments corresponded to few or several clones following digestion-amplification using either one or both MspI or HpaII. Unstable polymorphic fragments were common to at least 5 clones (E39/ HM83 marker 150 HpaII) up to 22 clones (E32/HM70 marker 116 HpaII) (Table 4).

\section{MSAP Marker Effectiveness}

The percentage of stable polymorphisms was calculated for all informative combinations. Combinations E35/HM70 and E45/HM83 produced the highest percentage of polymorphisms (9.21 and $5.49 \%$, respectively) compared to E32/HM70 and E39/HM46 being the least polymorphic ( 1.47 and $1.28 \%$, respectively) (Table 5).

The effectiveness of each MSAP primer combination was estimated using the PIC. PIC values were classed as "highly informative" (PIC >0.5), "reasonably informative" $(0.5>\mathrm{PIC}>0.25)$ and "slightly informative" 


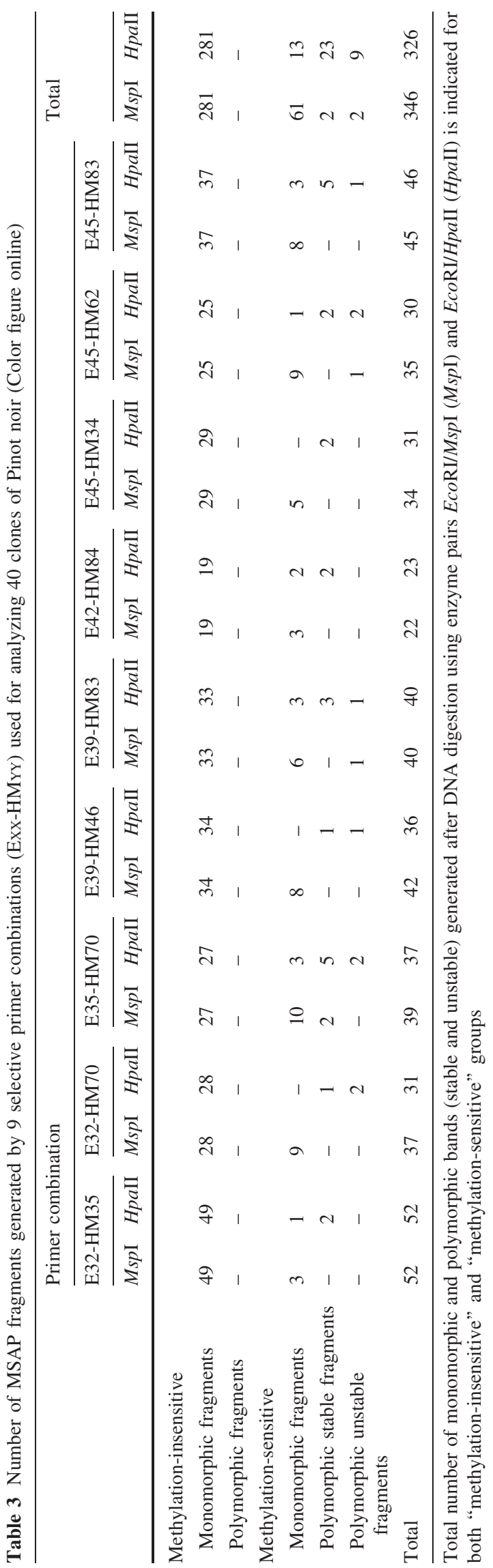

(PIC $<0.25$ ), as described by Botstein et al. [24]. Values were in agreement with the percentage of stable polymorphisms. An average PIC value of 0.54 was obtained for all informative MSAP combinations. E32/HM35, E35/HM70, E39/HM83, E42/HM84, E45/HM62 and E45/HM83 were the most informative primer pairs in this study, with E35/ HM70 (0.80) and E45/HM83 (0.81) displaying the highest PIC values. Only combination E45/HM34 (0.44) was considered as reasonably informative, while E39/HM46 $(0.22)$ and E32/HM70 (0.09) generated slightly informative PIC (Table 5).

Stable Polymorphisms and Clonal Discrimination

Since the presence/absence of unstable polymorphic fragments was not consistent among replicates (different plants from a given clone), they are not reliable for the distinction of individual clones. Therefore, only stable polymorphisms were used to produce individual MSAP profiles. Consequently, 38 profiles were obtained using the 9 informative primer combinations which generated a total of 23 stable polymorphic markers. The size of markers fluctuated between 109 and 573 base pairs (bp). Thirty-seven profiles were clone-specific, and only one MSAP profile was shared by more than one clone. Clones PN115, PN461 and PN583 displayed a common MSAP profile (profile 1). These clones could not be distinguished using the 9 informative combinations (Fig. 3).

Some informative markers were common to several Pinot noir clones; some were restricted to a few clones or to just one clone (Fig. 3). For example, the E32/H70-134 bp marker with $\mathrm{HpaII}$ was present for 38 out of 40 clones and was absent only for clones PN665 and PN668. Using primer combination E39/HM83, clones PN743 and PN778 were distinguished from the other clones by HpaII 345 and 130 bp fragments, respectively. E45/HM83-109 bp marker with HpaII was present only in clone PN111, and E35/ H70-180 bp marker was absent only in clone PN743 with HpaII (Fig. 3).

In addition, the primer combination E35/HM70 generated both MspI and HpaII polymorphisms for clones PN165 (marker 573 bp) and PN666 (marker 115 bp). With the same combination, only HpaII but not MspI marker 573 bp was absent only in clone PN927 and marker 115 bp in clone PN870 (Fig. 3).

Methylation-sensitive amplified polymorphism fragments were also scored for one clone of $V$. vinifera Syrah (SY) and one clone of $V$. vinifera Sangiovese (SG). The degree of polymorphism between varieties was 3-5 times higher as compared to that within the group of Pinot noir clones: $12.87 \%$ between PN/SY, $16.31 \%$ between PN/ SG, $17.83 \%$ between SY/SG versus $3.72 \%$ between all Pinot noir clones. 
Table 4 Number of Pinot noir clones showing unstable polymorphic fragments using enzyme pairs EcoRI/MspI (M) and EcoRI/HpaII $(\mathrm{H})$

\begin{tabular}{llll}
\hline Primer combination & $\begin{array}{l}\text { Fragment } \\
\text { size (bp) }\end{array}$ & $\begin{array}{l}\text { Restriction } \\
\text { enzyme }\end{array}$ & $\begin{array}{l}\text { Number of } \\
\text { clones }\end{array}$ \\
\hline E39-HM83 & 150 & M & 11 \\
E32-HM70 & 150 & H & 5 \\
E35-HM70 & 116 & H & 22 \\
& 124 & H & 13 \\
E39-HM46 & 293 & H & 10 \\
E45-HM62 & 122 & H & 12 \\
& 360 & H & 6 \\
& 166 & M & 13 \\
E45-HM83 & 166 & H & 13 \\
\hline
\end{tabular}

Table 5 MSAP primer pairs, number of fragments, percentage of stable polymorphisms and PIC values

\begin{tabular}{lclll}
\hline $\begin{array}{l}\text { Primer } \\
\text { combination }\end{array}$ & $\begin{array}{l}\text { Total } \\
\text { number of } \\
\text { fragments }\end{array}$ & $\begin{array}{l}\text { Number of } \\
\text { stable } \\
\text { polymorphisms }\end{array}$ & $\begin{array}{l}\text { \% of stable } \\
\text { polymorphisms }\end{array}$ & PIC \\
\hline E32-HM35 & 104 & 2 & 1.92 & $0.66^{\mathrm{a}}$ \\
E32-HM70 & 68 & 1 & 1.47 & $0.09^{\mathrm{c}}$ \\
E35-HM70 & 76 & 7 & 9.21 & $0.80^{\mathrm{a}}$ \\
E39-HM46 & 78 & 1 & 1.28 & $0.22^{\mathrm{c}}$ \\
E39-HM83 & 80 & 3 & 3.75 & $0.62^{\mathrm{a}}$ \\
E42-HM84 & 45 & 2 & 4.44 & $0.68^{\mathrm{a}}$ \\
E45-HM34 & 65 & 2 & 3.08 & $0.44^{\mathrm{b}}$ \\
E45-HM62 & 65 & 2 & 3.08 & $0.51^{\mathrm{a}}$ \\
E45-HM83 & 91 & 5 & 5.49 & $0.81^{\mathrm{a}}$ \\
Total & 672 & 25 & & 0.54 \\
Average & 75 & 2.78 & 3.75 & \\
\hline
\end{tabular}

PIC values are coded as "highly informative" (a), "reasonably informative" (b) and "slightly informative" (c) as described by Botstein et al. [24]

\section{Hierarchical Clustering Analysis}

Twenty-three MSAP informative markers were used to estimate epigenetic differences between all analyzed clones (40 V. vinifera Pinot noir). A dendrogram was developed (Fig. 4) using UPGMA.

Thirty-seven clones were individually distinguished. Only 3 clones remained clustered into one subgroup (PN115, PN461 and PN583).

The cluster analysis classified the 40 Pinot noir clones into seven major groups. Clones PN743 and PN870 were clearly separated by the first bifurcation subsequently followed by the group composed of clones PN777 and TF2. The third group consisted of four clones (PN389, PN665,
Fig. 3 Thirty-eight stable MSAP profiles generated by 9 selective primer combinations (EXX-HMYy) for 40 Pinot noir clones. Present (yellow) and absent (blue) stable MSAP fragments were obtained after amplification of digested DNA using restriction enzymes EcoRI/MspI (M) and EcoRI/HpaII (H). Corresponding clone(s) are indicated below each profile (Color figure online)

PN666 and PN668), followed by a fourth group formed of two clones (PN165 and PN927) and a fifth group with four clones (PN114, PN462, PN828 and PN871). The remaining clones were distributed in the two last groups composed of 16 and 10 clones, respectively.

Furthermore, some clones were separated by just one bifurcation representing a single polymorphism: PN114/ PN871, GL58/G2-13, PN111/TF1, PN113/PN374, PD79/ (PN583/PN115/PN461), PN386/F1 and PN375/PN388 (Fig. 4).

\section{Discussion}

Clonal propagation is used for numerous domesticated plants, such as banana, coffee, cassava, taro or grape, in order to maintain favorable genotypes [26]. In grapevine, $V$. vinifera clones are the result of somatic genetic/epigenetic alterations during plant development deriving from several cycles of vegetative propagation [2]. Phenotypic characteristics can be influenced by the environment and they not always make it possible to distinguish clones belonging to the same variety. PCR-based molecular markers are more environment-independent and more discriminating for evaluating intra-varietal diversity.

In the present study, epigenetic variability within a set of Pinot noir clones was assessed using the methylation-sensitive AFLP technique, MSAP. The AFLP technique has proven to be useful for intra-specific analysis, producing genome-wide anonymous dominant markers with highly reproducible patterns [27]. In the present study, DNA was extracted from 95 different plants from 40 Pinot noir clones, and after the 3 steps of MSAP (restriction-ligation, preamplification and selective amplification), very similar profiles were obtained. A total of 346 and 326 different MSAP fragments were scored after restriction with EcoRI/ MspI and with EcoRI/HpaII, respectively. The majority of these bands were monomorphic (i.e., present in all individual samples): 342 (98.8 \%) with EcoRI/MspI and 294 (90.2\%) with EcoRI/HpaII (Table 1).

DNA Methylation and Stability of MSAP Markers

An essential conclusion of the present study is the distinction of two types of MSAP polymorphisms in $V$. vinifera clones. On the one hand, we scored stable 

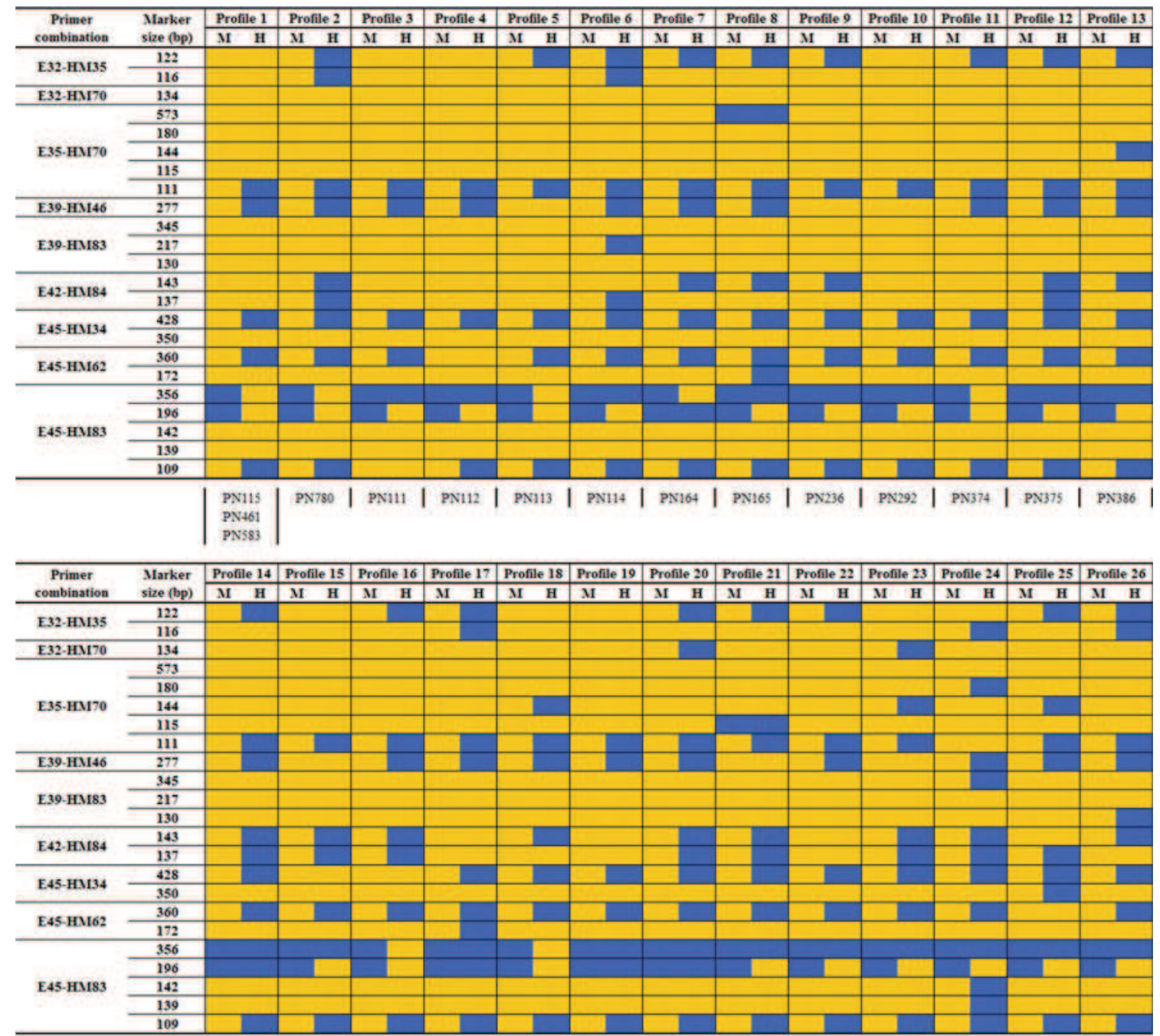

| pN388 | pN389 | pNa59 | pNa62 | pN521 | pN528 | pN665 | pN666 | pN667 | pN668 | pN743 | pN777 | pN778 |

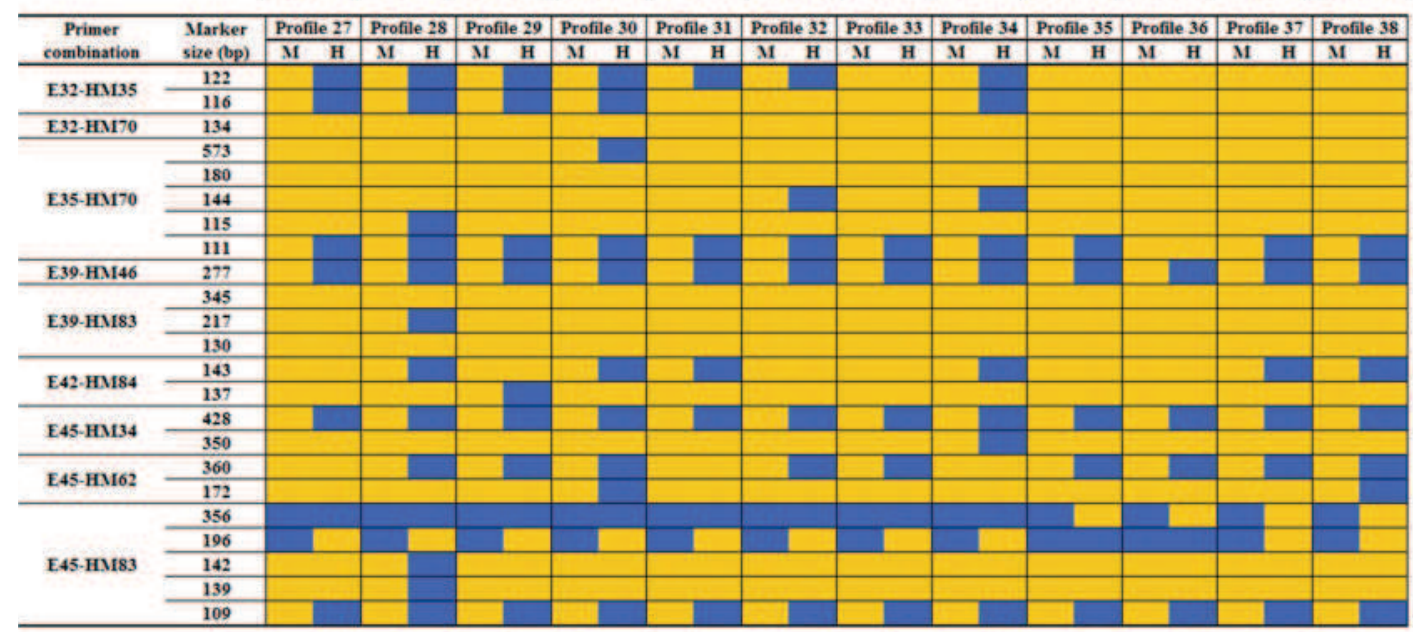

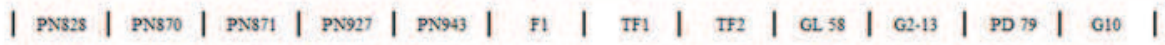

Presence of tragment

Absence of fragment 
polymorphisms since each clone showed a specific pattern which was exactly the same for the 2 or 5 plants per clone we have analyzed. In this case, different clones showed diverse stable MSAP profiles.

On the other hand, unstable fragments were amplified in one (or more) but not all plants from the same clone. Consequently, these fragments are not adequate for a robust distinction of Pinot noir clones. Some of these conserved fragments could be in fact stable fragments that were not always detected due to scoring difficulties influenced by technical problems (e.g., poor DNA quality, amplification artefacts) or mis-scoring as in the case of other PCR-based markers [28]. However, as these unstable fragments were detected in numerous individual plants, from 5 up to 22 clones depending on the detected fragment (Table 4), it is unlikely that they account for scoring errors in this study.

One great difference between genetic and epigenetic variation is that the second is less stable, can be altered by the environment and reversible [29-32]. Different profiles of methylation, which may be stable, unstable, heritable or lost, have been described in ecotypes of Arabidopsis [33].

Unstable polymorphisms, described here for the first time in grapevine, reflect alterations in the DNA methylation status of different individuals inside the same clone. As leaf samples were collected from plants in different field plots, detected unstable epigenetic variations could be the result of a diversity of agronomical and/or environmental conditions.

Epigenetic alterations are modulated by environmental factors, and the effects of stress may contribute to plant adaptation [32, 34]. Using MSAP, methylation profiles from different individuals grown in distinct environments were compared in some recent studies [35-37]. Epigenetic variation was greater than genetic variation and highly associated with environment. Therefore, epigenetic changes could contribute to the adaptation of plant populations to environmental changes. Yu et al. [37], for example, proposed that cytosine methylation alterations were induced by abiotic stresses in natural Leymus chinensis populations. Epigenetic alleles (epialleles) have been described in natural plant populations affecting important characters such as flower morphology [25]. DNA methylation changes triggered by environmental stresses can be passed on to the next generation [20, 38], and they could become fully or partially independent from genetic variations [39].

Epialleles might also have the faculty to return to their "wild forms" therefore becoming "metastable." This means that they are assumed to be stable due to a slow evolution process toward their "wild" form and not entirely due to a "true" stability [39]. However, as mentioned in Richards et al. [31], reversible and non-reversible components are included in epigenetic differentiation patterns among field populations measured in different

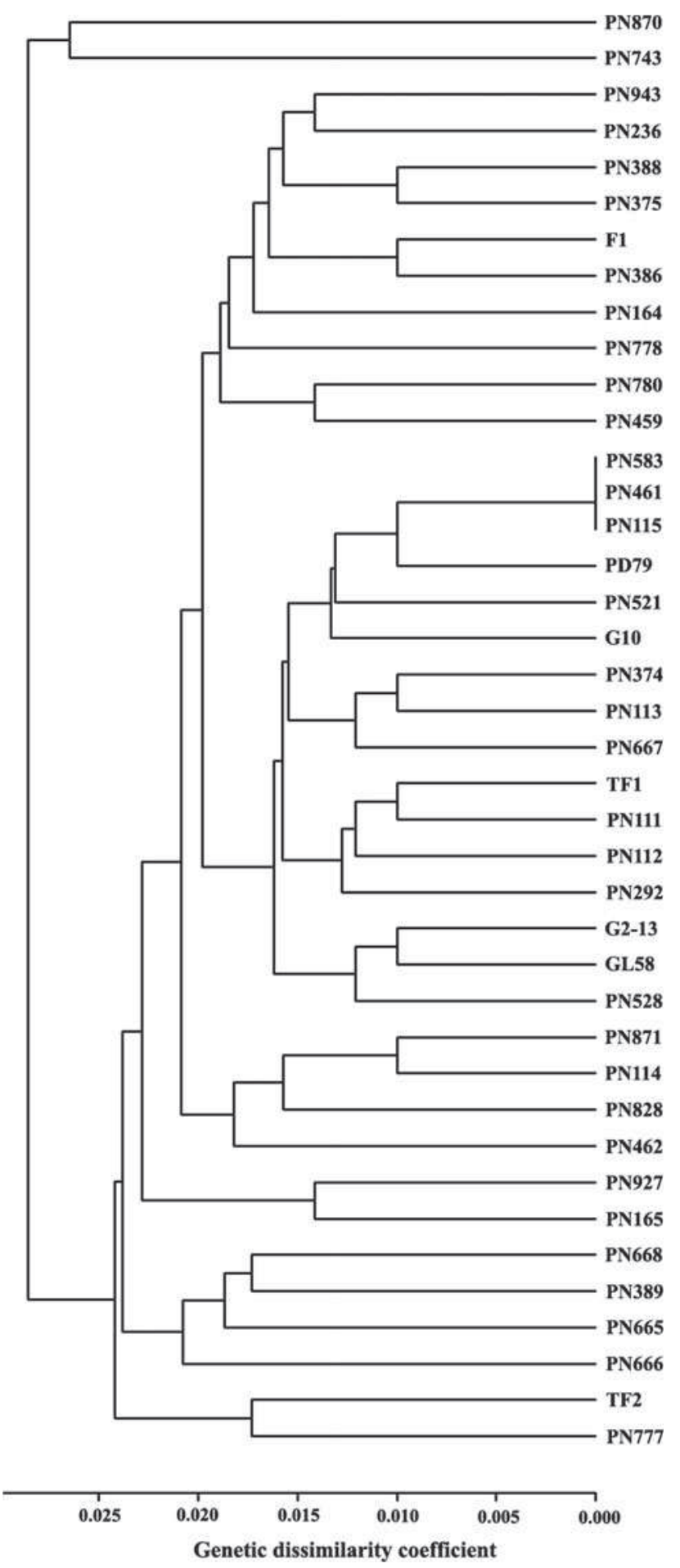

Fig. 4 Relationship between 40 Pinot noir clones as revealed by hierarchical cluster analysis of 23 stable MSAP polymorphic markers

environments. Hence, it is very likely that the different MSAP patterns obtained in our study are the result of spontaneous epimutations (stable and unstable) influenced by fluctuating environmental pressures and/or even cultural practices. 
The processes underlying epigenetic mutations (epimutations) among closely related individuals are still poorly understood. For instance, assessing DNA methylation could be very difficult since it might be variable within the same cell or even differing in a single cytosine at a specific location from one cell to another [40].

A more detailed analysis of the MSAP patterns brings information on the possible modifications in the cytosine methylation from one Pinot noir clone to another. In our experiments, we used two isoschizomers with different sensitivity to cytosine methylation, MspI and HpaII, both excising genomic DNA at CCGG sites [41, 42]. In most cases, stable and unstable polymorphisms we have identified correspond to fragments amplified after MspI restriction that are present or absent after HpaII restriction. If the fragment is amplified after restriction with both EcoRI/ MspI and EcoRI/HpaII, cytosines are unmethylated at the CCGG site. If the fragment is only amplified after restriction with EcoRI/MspI, the internal cytosine is methylated on both strands. Therefore, most stable and unstable polymorphisms correspond to differences in the methylation of the internal cytosine $\left(\mathrm{C}^{\mathrm{m}} \mathrm{CGG}\right)$ on both strands. This means that for a given polymorphic fragment, the CCGG site is unmethylated in some Pinot noir clones, whereas in others, the internal cytosines are methylated on both strands at the same restriction site.

With stable fragments, two other different situations were observed. In the first case, E35/HM70 marker 573 bp is absent for clone PN165 as well as marker $115 \mathrm{bp}$ for clone PN 666 after restriction with both EcoRI/MspI and EcoRI/HpaII. In addition, after restriction with EcoRI/ MspI, marker $573 \mathrm{bp}$ is present for clone PN927 and marker $115 \mathrm{bp}$ for clone PN870. For all the remaining clones, both markers (573 and $115 \mathrm{bp}$ ) are present after digestion with both pairs of restriction enzymes. Hence, the CCGG site of these fragments is either mutated in PN165 and PN666, or both internal and external cytosines are fully methylated ( ${ }^{\mathrm{m}} \mathrm{C}^{\mathrm{m}} \mathrm{CGG}$ on both strands). The second hypothesis is more likely since both fragments are present in all other Pinot noir clones.

In the second case, E45/HM83 fragments 356 and 196 bp were never amplified after restriction with EcoRI/ MspI and amplified in some clones after restriction with EcoRI/HpaII. As only HpaII can digest DNA at CCGG sites when the external cytosine is methylated on one strand (and not MspI), these polymorphisms correspond to differences in cytosine hemimethylation.

One of the disadvantages of the MSAP technique is that it underestimates the methylation level at the genome scale, since only CCGG restriction sites are targeted when using MspI and HpaII. Another complication of using MSAP lies upon the rare cutter EcoRI which is actually not entirely insensitive to methylation as overlapping of restriction sites might hamper correct cleavage during DNA digestion. In fact, EcoRI could be less active when restriction sites are methylated [20]. Sequencing analysis following bisulfite conversion of stable and unstable polymorphisms would be helpful to determine the methylation degree of each cytosine in the different fragments, also at EcoRI sites (GAATTC). This will allow the comparison between clones and/or individuals of the same clone and to verify the presence of different epialleles at a given locus in Pinot noir plants.

\section{Distinction of Pinot Noir Clones}

As stable fragments were present in all samples from the same clone, these polymorphisms are reliable for clonal identification. The informative stable markers detected in this research were either common to several Pinot noir clones, restricted to a few clones or just to one clone (Fig. 3). In total, 37 clones were distinguished. For each discriminated clone, a specific MSAP profile was obtained (Fig. 3).

Only one MSAP pattern corresponded to more than one clone. A single profile was shared by clones PN115, PN461 and PN583, all originally selected in the Côte d'Or region but registered in different years. For these 3 clones, it would be uncertain to assume traceability or identity errors during plant selection and/or multiplication, but these possibilities cannot be ruled out. Only further analysis and the generation of more polymorphic markers with additional primer combinations would allow distinguishing these clones. Since the total number of informative selective combinations used in this research was relatively moderate (9), it is very likely that by increasing their number, we would generate more discriminating stable markers and therefore distinguish multiple clones sharing identical MSAP profiles.

The dendrogram constructed after UPGMA cluster analysis using stable fragments ranked the 40 Pinot noir clones into seven major groups (Fig. 4). Methylation patterns were very similar between some clones because their distinction relied on a single polymorphism only. It is unlikely, however, that this represents any parental relationships among clones or possible linkages to common morphological traits.

\section{Epigenetic Markers Versus Genetic Markers}

Epigenetic and genetic variations can be very difficult to discern especially in highly diverse plant populations, as by definition, these populations exhibit extensive genetic variation [39]. Epigenetic variation could take place in a faster way compared to genetic variation, especially under constantly changing environmental conditions [15], and 
discriminating epigenetic from genetic differences represents a major challenge in population studies. Therefore, using the right genetic/epigenetic tools is necessary in order to characterize and exploit this variability.

As discussed by Richards [16], analyzing plant populations, for instance, clones generated by vegetative propagation would reduce genetic variation and allow scientists to focus on epigenetic differences. Consequently, grapevine could be an interesting plant model for such studies as it is commonly multiplied by vegetative propagation, thus generating clones genetically identical to the parent plant [2]. Clones are subsequently multiplied, and therefore, large populations corresponding to the same clone are subjected to a variety of field and garden conditions.

Microsatellite markers are very powerful for management of grapevine germplasm banks [8] and cultivar identity [43]. Nevertheless, previous studies have shown that they are not suitable for studying intra-clonal variability. For example, most of the SSRs did not show differences among grapevine clones and they were either not very powerful $[7,8]$, although others partially revealed diversity in grapevine collections [5, 6].

In our study, MSAP allowed the intra-varietal distinction of Pinot noir clones. Previous studies have shown that epigenetic variation detected by MSAP was greater than the genetic variation detected by AFLP for separating abnormal and normal-intermediate flower phenotypes in Solanum ruiz-lealii [25]. In grapevine, Schellenbaum et al. [19] used MSAP to study somaclonal variation in Syrah and Chardonnay cultivars allowing the identification of methylation alterations and possible methylation hotspots.

Other strategies have involved more than one technique to be able to generate enough marker information. For example, Castro et al. [44] used IRAP (interretrotransposon amplified polymorphism), REMAP (retrotransposon-microsatellite amplified polymorphism), S-SAP (sequence-specific amplification polymorphism) and AFLP techniques to discriminate a set of $41 \mathrm{~V}$. vinifera cultivars, including 37 clones within 6 of them. For clonal identification, IRAP did not produce any polymorphism, while REMAP allowed the distinction of only 3 clones. AFLP and S-SAP polymorphic markers discriminated 15 and 9 clones, respectively.

Recently, using next-generation sequencing technology, portions of the genome of 3 Pinot noir clones were compared [3]. Insertion polymorphisms accounted as the major cause of genetic differences between the 3 clones. Using primers derived from four types of mobile elements, specific S-SAP patterns were obtained for 60 Pinot clones, including 40 Pinot noir clones, although no plant replicates were studied. Moreover, caution must be taken since the detection of polymorphisms by S-SAP strongly depends on the activity of mobile elements [3]. This method may produce different profiles from one plant to another within a given clone, and therefore, it could be inadequate for the distinction of grapevine clones.

Meneghetti et al. [45] proposed a protocol for studying intra- and inter-varietal genetic variability using a combination of SSRs, AFLPs, SAMPLs (selective amplification of microsatellite polymorphic loci), M-AFLP (microsatellite-AFLP) and ISSR (inter-simple sequence repeat) in $243 \mathrm{~V}$. vinifera accessions corresponding to 7 cultivars establishing genetic differences and correlating them to specific geographic regions or morphological traits. The reason behind this correlation was not entirely discussed as mentioned by the authors though it seems that human selection played an important role [45]. For example, in Malvasia nera di Brindisi/Lecce, Primitivo and Negroamaro cultivars, all largely cultivated in the Apulia region in Italy, spread seemed to be limited due to traditional propagation practices therefore making it possible to establish possible links between genetic differences and geographic origins [46].

The MSAP approach using stable epigenetic markers could be also a complementary tool to other PCR-derived methods, as used by several authors [3, 7, 9-12, 45, 46].

\section{General Conclusion}

Using the MSAP technique, 37 out of 40 Pinot noir clones were discriminated using only 9 discriminating primer combinations. Only 3 clones could not be distinguished, and they remained clustered in one subgroup. Difference in full methylation of internal cytosine $\left(\mathrm{C}^{\mathrm{m}} \mathrm{CGG}\right)$ was identified as the major source of variation detected by MSAP. The detected polymorphic fragments were scored as stable and unstable polymorphisms.

This study underlies the importance of epigenetic approaches and represents a major contribution in the evaluation of Pinot noir clonal diversity. The identification of stable epigenetic markers in Pinot noir could be suitable for the development of straightforward techniques for clonal identification. Such techniques could potentially accelerate the selection process in plant nurseries and might become useful for management of germplasm collections and for routine analysis when certainty of plant identity is needed.

Acknowledgments The authors would like to thank Jean-Michel Menant (ATVB) and Francis Minet (Pepinières Guillaume) for kindly providing the plant material used in this study, Dr. Najoi El Azhari (Welience) for technical support with the Li-Cor sequencer, Franck Fickinger-Villemin for MSAP technical assistance and Dr. Marc Lollier for the statistical part of the manuscript. This study was funded by the Fonds Unique Interministériel (FUI) and OSEO including a $\mathrm{PhD}$ grant for J. O. 


\section{References}

1. Rives, M. (1961). Bases génétiques de la sélection clonale chez la vigne. Annals of Amélior Plantes, 11, 337-348.

2. Pelsy, F. (2010). Molecular and cellular mechanisms of diversity within grapevine varieties. Heredity, 104, 331-340.

3. Carrier, G., Le Cunff, L., Dereeper, A., Legrand, D., Sabot, F., Bouchez, O., et al. (2012). Transposable elements are a major cause of somatic polymorphism in Vitis vinifera L. PLoS One, 7, e32973.

4. Riaz, S., Garrison, K. E., Dangl, G. S., Boursiquot, J.-M., \& Meredith, C. P. (2002). Genetic divergence and chimerism within ancient asexually propagated winegrape cultivars. Journal of the American Society for Horticultural Science, 127, 508-514.

5. Pelsy, F., Hocquigny, S., Moncada, X., Barbeau, G., Forget, D., Hinrichsen, P., et al. (2010). An extensive study of the genetic diversity within seven French wine grape variety collections. Theoretical and Applied Genetics, 120, 1219-1231.

6. Cipriani, G., Spadotto, A., Jurman, I., Di Gaspero, G., Crespan, M., Meneghetti, S., et al. (2010). The SSR-based molecular profile of 1005 grapevine (Vitis vinifera L.) accessions uncovers new synonymy and parentages, and reveals a large admixture amongst varieties of different geographic origin. Theoretical and Applied Genetics, 121, 1569-1585.

7. Imazio, S., Labra, M., Grassi, F., Winfield, M., Bardini, M., \& Scienza, A. (2002). Molecular tools for clone identification: The case of the grapevine cultivar "Traminer". Plant Breed, 121, $531-535$.

8. Laucou, V., Lacombe, T., Dechesne, F., Siret, R., Bruno, J.-P., Dessup, M., et al. (2011). High throughput analysis of grape genetic diversity as a tool for germplasm collection management. Theoretical and Applied Genetics, 122, 1233-1245.

9. Labra, M., Imazio, S., Grassi, F., Rossoni, M., \& Sala, F. (2004). Vine1 retrotransposon-based sequence-specific amplified polymorphism for Vitis vinifera L. genotyping. Plant Breed, 123, 180-185.

10. Blaich, R., Konradi, J., Rühl, E., \& Forneck, A. (2007). Assessing genetic variation among Pinot noir (Vitis vinifera L.) clones with AFLP markers. American Journal of Enology and Viticulture, 58, 526-529.

11. Anhalt, U. C., Martínez, S. C., Rühl, E., \& Forneck, A. (2011). Dynamic grapevine clones-an AFLP-marker study of the Vitis vinifera cultivar Riesling comprising 86 clones. Tree Genetics and Genomes, 7, 739-746.

12. Wegscheider, E., Benjak, A., \& Forneck, A. (2009). Clonal variation in Pinot noir revealed by S-SAP involving universal retrotransposon-based sequence. American Journal of Enology and Viticulture, 60, 104-109.

13. Verriès, C., Bès, C., This, P., \& Tesnière, C. (2000). Cloning and characterization of Vine-1, a LTR-retrotransposon-like element in Vitis vinifera L., and other Vitis species. Genome, 43, 366-376.

14. Kaeppler, S. M., Kaeppler, H. F., \& Rhee, Y. (2000). Epigenetic aspects of somaclonal variation in plants. Plant Molecular Biology, 43, 179-188.

15. Paun, O., Bateman, R. M., Fay, M. F., Hedre, M., Civeyrel, L., \& Chase, M. W. (2010). Stable epigenetic effects impact adaptation in allopolyploid orchids (Dactylorhiza: Orchidaceae). Molecular Biology and Evolution, 27, 2465-2473.

16. Richards, E. J. (2011). Natural epigenetic variation in plant species: A view from the field. Current Opinion in Plant Biology, 14, 204-209.

17. Fang, J.-G., \& Chao, C. T. (2007). Methylation-sensitive amplification polymorphism in date palms (Phoenix dactylifera $\mathrm{L}$.) and their off-shoots. Plant Biology, 9, 526-533.

18. Monteuuis, O., Doulbeau, S., \& Verdeil, J.-L. (2008). DNA methylation in different origin clonal offspring from a mature Sequoiadendron giganteum genotype. Trees, 22, 779-784.
19. Schellenbaum, P., Mohler, V., Wenzel, G., \& Walter, B. (2008). Variation in DNA methylation patterns of grapevine somaclones (Vitis vinifera L.). BMC Plant Biology, 8, 78.

20. Verhoeven, K. J. F., van Dijk, P. J., \& Biere, A. (2010). Changes in genomic methylation patterns during the formation of triploid asexual dandelion lineages. Molecular Ecology, 19, 315-324.

21. Japelaghi, R. H., Haddad, R., \& Garoosi, G.-A. (2011). Rapid and efficient isolation of high quality nucleic acids from plant tissues rich in polyphenols and polysaccharides. Molecular Biotechnology, 49, 129-137.

22. Schellenbaum, P., Walter, B. and Maillot, P. (2011) Is DNA methylation a marker of somaclonal variation induced by in vitro culture?, in Plant tissue culture and applied plant biotechnology, Editors Kumar, A. and Roy, S. Aaviskar Publishers, New Delhi, India: pp. 17-63 (ISBN: 9788179103630).

23. Fox, J. (2005). The R Commander: A basic-statistics graphical user interface to R. Journal of Statistical Software, 14, 1-42.

24. Botstein, D., White, R. L., Skolnick, M., \& Davis, R. W. (1980). Construction of a genetic linkage map in man using restriction fragment length polymorphisms. American Journal of Human Genetics, 32, 314-331.

25. Marfil, C. F., Camadro, E. L., \& Masuelli, R. W. (2009). Phenotypic instability and epigenetic variability in a diploid potato of hybrid origin, Solanum ruiz-lealii. BMC Plant Biology, 9, 21.

26. McKey, D., Elias, M., Pujol, B., \& Duputié, A. (2010). The evolutionary ecology of clonally propagated domesticated plants. New Phytologist, 186, 318-332.

27. Meudt, H. M., \& Clarke, A. C. (2007). Almost forgotten or latest practice? AFLP applications, analyses, and advances. Trends in Plant Science, 12, 106-117.

28. Pompanon, F., Bonin, A., Bellemain, E., \& Taberlet, P. (2005). Genotyping errors: Causes, consequences and solutions. Nature Reviews Genetics, 6, 847-859.

29. Kalisz, S., \& Purugganan, M. D. (2004). Epialleles via DNA methylation: Consequences for plant evolution. Trends in Ecology \& Evolution, 19, 309-314.

30. Reinders, J., Wulff, B. B. H., Mirouze, M., Marí-Ordóñez, A., Dapp, M., Rozhon, W., et al. (2009). Compromised stability of DNA methylation and transposon immobilization in mosaic Arabidopsis epigenomes. Gene Development, 23, 939-950.

31. Richards, C. L., Bossdorf, O., \& Verhoeven, K. J. F. (2010). Understanding natural epigenetic variation. New Phytologist, $187,562-564$

32. Grativol, C., Hemerly, A. S., \& Ferreira, P. C. (2012). Genetic and epigenetic regulation of stress responses in natural plant populations. Biochimica et Biophysica Acta, 1819, 176-185.

33. Vaughn, M. W., Tanurdžić, M., Lippman, Z., Jiang, H., Carrasquillo, R., Rabinowicz, P. D., et al. (2007). Epigenetic natural variation in Arabidopsis thaliana. PLoS Biology, 5, 1617-1629.

34. Feil, R., \& Fraga, M. F. (2012). Epigenetics and the environment: Emerging patterns and implications. Nature Reviews Genetics, 13, 97-109.

35. Herrera, C. M., \& Bazaga, P. (2010). Epigenetic differentiation and relationship to adaptive genetic divergence in discrete populations of the violet Viola cazorlensis. New Phytologist, 187, 867-876.

36. Lira-Medeiros, C. F., Parisod, C., Fernandes, R. A., Mata, C. S., Cardoso, M. A., \& Ferreira, P. C. G. (2010). Epigenetic variation in mangrove plants occurring in contrasting natural environment. PLoS One, 5, e10326.

37. Yu, Y., Yang, X., Wang, H., Shi, F., Liu, Y., Liu, J., et al. (2013). Cytosine methylation alteration in natural populations of Leymus chinensis induced by multiple abiotic stresses. PLoS One, 8, e55772.

38. Becker, C., \& Weigel, D. (2012). Epigenetic variation: Origin and transgenerational inheritance. Current Opinion in Plant Biology, 15, 562-567. 
39. Schmitz, R. J., \& Ecker, J. R. (2012). Epigenetic and epigenomic variation in Arabidopsis thaliana. Trends in Plant Science, 17, 149-154.

40. Feng, S., \& Jacobsen, S. E. (2011). Epigenetic modifications in plants: An evolutionary perspective. Current Opinion in Plant Biology, 14, 179-186.

41. Reyna-Lopez, G. E., Simpson, J., \& Ruiz-Herrera, J. (1997). Differences in DNA methylation patterns are detectable during the dimorphic transition of fungi by amplification of restriction polymorphisms. Molecular and General Genetics, 253, 703-710.

42. Xiong, L. Z., Xu, C. G., Saghai Maroof, M. A., \& Zhang, Q. (1999). Patterns of cytosine methylation in an elite rice hybrid and its parental lines, detected by a methylation-sensitive amplification polymorphism technique. Molecular and General Genetics, 261, 439-446.

43. This, P., Jung, A., Boccacci, P., Borrego, J., Botta, R., Costantini, L., et al. (2004). Development of a standard set of microsatellite reference alleles for identification of grape cultivars. Theoretical and Applied Genetics, 109, 1448-1458.

44. Castro, I., D’Onofrio, C., Martín, J. P., Ortiz, J. M., De Lorenzis, G., Ferreira, V., et al. (2012). Effectiveness of AFLPs and retrotransposon-based markers for the identification of Portuguese grapevine cultivars and clones. Molecular Biotechnology, 52, 26-39.

45. Meneghetti, S., Calò, A., \& Bavaresco, L. (2012). A strategy to investigate the intravarietal genetic variability in Vitis vinifera L. for clones and biotypes identification and to correlate molecular profiles with morphological traits or geographic origins. Molecular Biotechnology, 52, 68-81.

46. Meneghetti, S., Costacurta, A., Morreale, G., \& Calò, A. (2012). Study of intra-varietal genetic variability in grapevine cultivars by PCR-derived molecular markers and correlations with the geographic origins. Molecular Biotechnology, 50, 72-85. 